\title{
Tracheal Stoma Necrosis: A Case Report
}

\author{
Stella Pak, MD \\ Arjan Flora, MD \\ Young-Sook Yoon, MD \\ Department of Medicine \\ University of Toledo Medical Center \\ Toledo, $\mathrm{OH}, \mathrm{USA}$
}

\begin{abstract}
Acute tracheal dilatation, due to an overinflated cuff, has been reported early in the course of mechanical ventilation through an endotracheal tube. Tracheal stoma necrosis is a rare complication, but such can accompany acute tracheal dilation. Herein, we report a case of tracheal necrosis 9 days following tracheostomy placement in a 71year old woman associated with overinflation of the tracheal tube cuff. This case report aims to 1) add to the scant body of knowledge about the diagnosis and management for the patients with tracheal stoma necrosis and 2) raise awareness for error-traps in interpreting diagnostic images, specifically satisfaction of search error, inattentional blindness error, and alliterative error.
\end{abstract}

\section{Case Report}

A 71-year-old woman with a history of chronic respiratory failure on mechanical ventilation presented to the emergency department for bleeding around the tracheostomy site. The tracheostomy was recently inserted 9 days prior to admission. A chest radiograph demonstrated left lower lobe atelectasis, pleural effusion, and cardiomegaly that was consistent with pre-existing congestive heart failure (Figure 1).

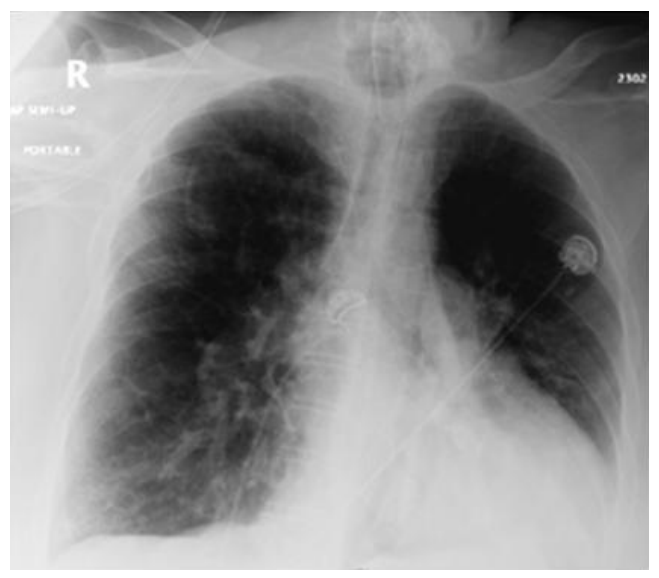

Figure 1. Chest radiograph (AP) performed during first admission. 
The cuff overinflation was demonstrated as a spherical shaped hypolucent region surrounding the trachea. However, the lesion escaped attention possibly because the focus of attention was limited to the thoracic compartment. A CT of the soft tissue in the neck ruled out the possibility of hematoma or infection. However, the features suggestive of overinflation of tracheostomy tube once again escaped attention. The spherical shaped hypolucent area, representing the cuff, was $3.9 \mathrm{~cm}$ in the anteriorposterior axis and $3.8 \mathrm{~cm}$ along the right-left axis.

A fiberoptic bronchoscopy through the tracheostomy tube revealed a large blood clot obstructing the distal end of the tube. A necrotic lesion around the stoma was also found. Careful observation via the bronchoscope during the procedure revealed no tearing or rupture. The patient was conservatively treated with vancomycin and cefepime for treatment of a ventilator-associated pneumonia. The oozing of blood from the tracheostomy stopped on with conservative wound care, including cleaning and dressing. She returned back to her baseline and was subsequently discharged on $3^{\text {rd }}$ day of admission. During this first admission, a tracheostomy tube exchange was not done due to bleeding from the stoma.

The patient was readmitted 12 days after discharge for an episode of hematemesis of approximately $400 \mathrm{~mL}$ of bright red blood. A chest radiograph showed satisfactory position of tracheotomy tube and cardiomegaly at baseline (Figure 2).

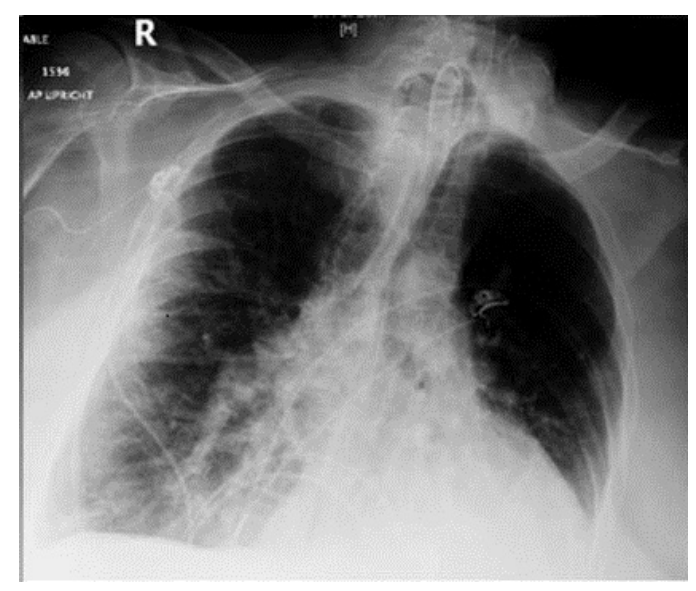

Figure 2. Chest radiograph (AP) after readmission.

For the third time, the features suggestive of cuff-overinflation went unnoticed, delaying accurate diagnosis and proper treatment.

As a part of the patient's evaluation, a CT of the chest with intravenous contrast was done, revealing the overinflated cuff of the trachea tube into the soft tissue of the neck (Figure 3). The ovoid shaped hypolucent area, representing the cuff, was $5.3 \mathrm{~cm}$ in the anterior-posterior axis and $4.6 \mathrm{~cm}$ along the right-left axis. 


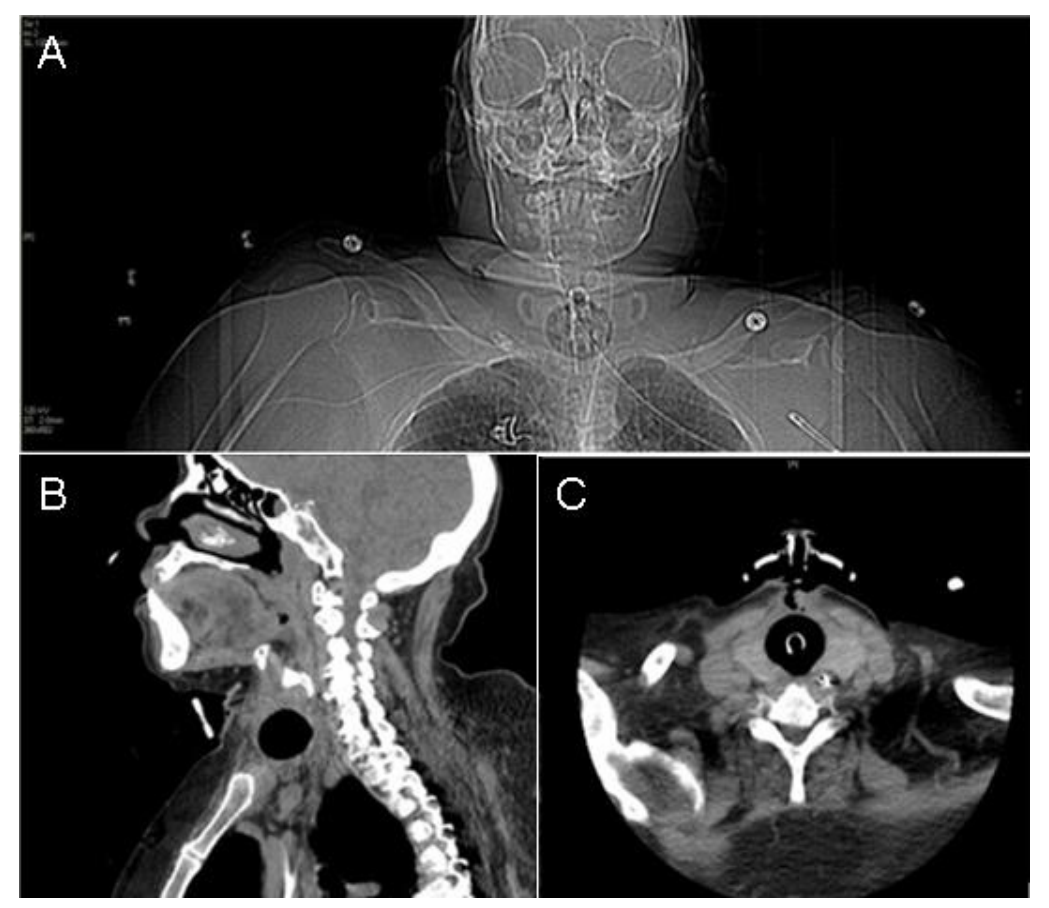

Figure 3. Thoracic CT scan showing the overinflated tracheostomy cuff in the (A) coronal, (B) sagittal, and (C) axial views.

The Shiley proximal tracheal tube was urgently replaced with a portex Bivona tracheal tube. The new tracheostomy tube is more extensible, soft, and longer in distal length. Postoperatively, the patient was kept ventilated in the ICU. Repeated chest CT showed the new tracheostomy tube in satisfactory position and normalization of trachea shape. She made an uneventful recovery and was discharged 8 days after the tracheotomy tube replacement.

\section{Discussion}

A case of nonfatal hemorrhage due to innominate artery erosion with soft tissue necrosis at the stoma site of a tracheostomy is presented. In this ventilator-dependent patient with a recent tracheotomy stoma creation, an overinflated cuff of a tracheotomy tube was the key culprit in the pathology. Tracheal tube cuff pressure should be monitored so that it does not exceed a reasonable estimate of capillary perfusion pressure. Cuffs with pressure over $25 \mathrm{mmHg}$ can compress the surrounding soft tissue, including delicate vascular structures. The damage to the vasculature in contact with the tube can result in ischemic necrosis in the soft tissue. If left untreated, these necrotic regions can develop infection or undergo fibrosis, leading to progressive stenosis (1).

A number of cognitive errors led to multiple episodes of misdiagnosis in this patient. Satisfaction of search error is a type of false negative error caused by premature 
termination of search after an abnormality has been detected (2). In this patient, we readily detected several abnormalities-cardiomegaly, pulmonary atelectasis, and pleural effusion. These initial findings likely led us to subconsciously neglect later findings.

Inattentional blindness error is a false negative error caused by the psychological lack of attention on an unexpected stimulus (3). In the present case, none of the diagnostic imaging was taken to check for cuff-overinflation. The images from the first admission were ordered for a concern of NG tube malposition, infection, and hematoma. The images ordered during the second admission were ordered to check the tracheotomy tube position. The thoracic compartment (the area for the expected abnormalities) received a disproportionately large amount of attention, whereas only a scant amount of attention was paid to the neck compartment.

Alliterative error is an error caused by a preconceived notion from a previous interpretation by a colleague or oneself (4). The negative finding in the previous reports could have affected the subsequent interpretative performance.

To the best of our knowledge, there are only 3 other cases of soft tissue necrosis caused by cuff overinflation. In two of these cases, the extended trachea did not recoil back to the previous size $(5,6)$. In the presented case, the stretched trachea recoiled back, similar to the case described by Sachdeva and his colleagues (7). The prognostic value of this difference in recovery is unknown, but might have a significant clinical implication. To explore the clinical relevance of this finding, more data on this condition is needed.

\section{Teaching Points}

1. Careful attention should be paid to cuff inflation pressure in patients presenting with bleeding at the tracheostomy site.

2. Conscious efforts to avoid well-known errors in diagnostic image interpretation, such as satisfaction of search error, and inattentional blindness error, should be made to improve diagnostic accuracy.

\section{References}

1. De Leyn P, Bedert L, Delcroix M, et al. Tracheotomy: clinical review and guidelines. Eur J Cardiothorac Surg. 2007 Sep;32(3):412-21. [CrossRef] [PubMed]

2. Ashman CJ, Yu JS, Wolfman D. Satisfaction of search in osteoradiology. AJR Am J Roentgenol. 2000 Aug;175(2):541-4. [CrossRef] [PubMed]

3. Richards A, Hannon EM, Derakshan N. Predicting and manipulating the incidence of inattentional blindness. Psychol Res. 2010 Nov;74(6):513-23. [CrossRef] [PubMed] 
4. Berlin L. Malpractice issues in radiology. Alliterative errors. AJR Am J Roentgenol. 2000 Apr;174(4):925-31. [CrossRef] [PubMed]

5. Rhodes A, Lamb FJ, Grounds RM, Bennett ED. Tracheal dilatation complicating tracheal intubation. Anaesthesia. 1997 Jan;52(1):70-2. [CrossRef] [PubMed]

6. Honig EG, Francis PB. Persistent tracheal dilatation: onset after brief mechanical ventilation with a "soft-cuff" endotracheal tube. South Med J. 1979 Apr;72(4):487-90. [CrossRef] [PubMed]

7. Sachdeva A, Pickering EM, Reed RM, Shanholtz CB. Ice cream cone sign: reversible ballooning of the trachea due to tracheostomy tube cuff overinflation. BMJ Case Rep. 2016 May 4;2016. [CrossRef] [PubMed] 